\title{
Flowering Biological Characteristics of Camellia weiningensis Y.K. Li.
}

\author{
Hongli Wei, Chao Gao, Jie Qiu, Li Long, Biao Wang, Lu Yang, and \\ Yang Hu \\ Institute for Forest Resources and Environment of Guizhou, Key Laboratory \\ of Forest Cultivation in Plateau Mountain of Guizhou Province, College of \\ Forestry, Guizhou University, Guiyang 550025, China
}

Additional index words. floral organ, flowering biology, pollen, pollination, germination rate

\begin{abstract}
This study aimed to investigate the flowering biological characteristics, floral organ characteristics, and pollen morphology of Camellia weiningensis Y.K. Li. These features of adult $C$. weiningensis plants were observed via light microscopy and scanning electron microscopy (SEM). Pollen viability and stigma receptivity were detected using 2,3,5-triphenyltetrazole chloride (TTC) staining and the benzidine-hydrogen peroxide reaction method. $C$. weiningensis is monoecious, with alternate leaves and glabrous branchlets. Its flowering period lasts 2 to 4 months, and the flowering time of individual plants lasts $\approx \mathbf{5 0}$ days, with the peak flowering period from the end of February to the middle of March. It is a "centralized flowering" plant that attracts a large number of pollinators. Individual flowers are open for 12 to 13 days, mostly between 1230 and $1630 \mathrm{HR}$, and include four to six sepals, six to eight petals, $\approx 106$ stamens, an outer ring of $\approx \mathbf{2 4 . 6}$-mm-long stamens, an inner ring of $\approx \mathbf{1 3 . 4}$-mm-long stamens, one pistil, and nine to 12 ovules. The flowers are light pink. The style is two- to three-lobed and $16.6 \mathrm{~mm}$ long, showing a curly "Y" shape. The contact surface of the style is covered with papillary cells and displays abundant secretory fluid and a full shape, facilitating pollen adhesion. The pollen is rhombohedral cone-shaped, and there are germ pores (tremoids). The groove of the germ pore is slender and extends to the two poles (nearly reaching the two poles). The pollen is spherical in equatorial view and trilobate in polar view. The pollen vitality was highest at the full flowering stage, and the stigma receptivity was greatest on days 2 to 3 of flowering. The best concentration of sucrose medium for pollen germination was $100 \mathrm{~g} / \mathrm{L}$. The number of pollen grains per anther was $\approx 2173$, and the pollen-to-ovule ratio was 23,034:1. $C$. weiningensis is cross-pollinated. Seventy-two hours after cross-pollination, the pollen tube reached the base, and a small part entered the ovary. The time when the pollen tube reached the base after pollination was later than that in commonly grown Camellia oleifera. The results of this study might lay an important foundation for the flowering management, pollination time selection, and cross-breeding of $C$. weiningensis.
\end{abstract}

In higher plants, flowering marks the transition from vegetative growth to reproductive growth and determines the critical period and quality of plant reproductive growth. Studies on plant phenology are of great practical significance for understanding the reproductive growth of plants (Wright and Meagher, 2003). The flowering biology of a plant includes multiple variables. For example, the comprehensive

Received for publication 26 May 2021. Accepted for publication 23 July 2021.

Published online 27 September 2021.

This work was supported by the Science and Technology Planning Projects of Guizhou (Qian $\mathrm{Ke} \mathrm{He}[2019] 2310$ ), Science and Technology Project of Guizhou Education Department (Qian jiao He KY [2018]097), National Natural Science Foundation of China (31800516, 32060331), Cultivation Project of Guizhou University ([2019]35) and Research Project of Introducing Talents in Guizhou University ([2017]41).

We thank Jiayin Zhi for his help in sampling.

C.G. is the corresponding author. E-mail: gaochao@gzu.edu.cn.

This is an open access article distributed under the CC BY-NC-ND license (https://creativecommons. org/licenses/by-nc-nd/4.0/). phenological indexes of individual plants, such as the initial flowering stage, full flowering stage, and final flowering stage, which constitute plant phenology, are regulated by both external factors (e.g., climatic conditions and hormones) and internal factors (e.g., gene inheritance). The successful reproduction, mating system, and genetic diversity of plant populations are affected by the comprehensive characteristics of flowers, including the floral morphology, flowering phenology, and inflorescence characteristics (Harder and Barrett, 1995; Richards et al., 1986; Wyatt et al., 1982). The floral pattern mainly includes the number of flowers open at a certain time and the arrangement of the flowers (Barrett, 2003; Harder and Barrett, 1995; Mitchell et al., 2004).

In the production and cultivation of $C$. oleifera, poor performance, including severe flower and fruit dropping and low yields, often occurs, which greatly affects the longterm efficient and stable development of the C. oleifera industry. Therefore, improving the fruit setting rate and ensuring fruit yields have been the top priorities in C. oleifera production in recent years. Since the 1850 s, researchers in China have been conducting studies on the protection of flowers and fruits in terms of water and fertilizer management and pest control, but little effect has been produced. The flowering of $C$. oleifera occurs mostly in cold seasons (autumn and winter), and insects are required for cross-pollination. When the temperature during the flowering period is low, insects do not move frequently, and the floral organs are prone to frostbite, which seriously hinders pollen germination, pollination, and fertilization. Plant flowering biology is the basis of plant reproduction. Conducting studies on flowering biological characteristics is of great significance for revealing temporal selection that affects the flowering order of plants and reproduction among individual species.

Mountainous regions are extreme environments for plant growth, and their low temperatures, windy weather, and strong radiation have great impacts on the flowering characteristics, breeding system, and pollination process of plants (Bingham and Orthner, 1998; Kudo and Suzuki, 2002; Molau, 1993; Scheffknecht et al., 2007). To date, numerous studies on the flowering habits of mountainous plants in Europe, Africa, and North and South America (Bingham and Orthner, 1998; Korner, 1999; Molau, 1993; Totland, 1997; Egisdóttir and Thórhallsdóttir, 2006), as well as on the Tibetan Plateau of Asia (Duan et al., 2005; Tsukaya et al., 2002), have been performed. These studies show that the flowering phenological characteristics of alpine plants are closely related to their poor ecological environment. Research on the flowering phenological characteristics of alpine plants can provide a theoretical basis for revealing the breeding system and genetic breeding of angiosperms, which will further guarantee the breeding success of these species, thereby increasing the species continuity.

C. weiningensis Y.K. Li. belongs to the Camellia L. genus of the Theaceae family and is a wild woody oil plant growing at 1800 to 2700 -m altitudes in Weining County, Guizhou, China. C. weiningensis was first discovered by Wang et al. (1979) in 1977 and was named by Associate Professor Yongkang Li. Later, it was classified as Sect Brhvistyla chang sect.n of the Camellia L. genus of the Theaceae family by Professor Hongda Zhang from Sun Yat-sen University. C. weiningensis is an endemic economic forest species, important woody oil tree species, and ecologically protected tree species in the Guizhou mountainous area, which is an important location in the current targeted poverty alleviation plan for the Weining area. C. weiningensis bears great similarities to common $C$. oleifera. C. weiningensis oil softens blood vessels and therefore is beneficial for populations with hyperlipidemia and hypertension. C. weiningensis is distributed at high altitudes and is characterized by cold and drought resistance, strong soil preferences, early fruit maturity, thin fruit and seed shells, a high kernel yield, and a high oil content; furthermore, its oil is bright and clear. It is the first choice of tree species for rural revitalization and the 
development of woody oil production in highaltitude, cold areas of Guizhou Province. Since its discovery 40 years ago, a number of studies on $C$. weiningensis have been reported. However, all of these studies focused on the resource status, oil quality, and economic characteristics of $C$. weiningensis. To the best of our knowledge, no observational study of its flowering phenology has been carried out. Similar to ordinary $C$. oleifera, $C$. weiningensis also displays serious flower- and fruitfall, a low fruit setting rate, and a low yield. C. weiningensis lacks basic theoretical research, which affects its development and utilization.

In this study, the flowering biology (i.e., flowering phenology, individual-flower opening process, floral organ morphological characteristics, pollen activity, pollen quantity, pollen-to-ovule $(\mathrm{P} / \mathrm{O})$ ratio, pollen morphological characteristics, and pollen tube growth) of $C$. weiningensis was examined. The purpose of this study was to provide a theoretical basis for research on the flowering mechanism and hybrid breeding of $C$. weiningensis, and the results might deepen the understanding of the production and cultivation of $C$. weiningensis.

\section{Materials and Methods}

Experimental materials. All materials used in this experiment were from $C$. weiningensis plants with healthy growth and normal blossoming. The experimental site is located at the C. oleifera Research Station of Guizhou University, Tujie Town, Weining County, Bijie City, Guizhou Province, China (lat. $27^{\circ} 11^{\prime}$ $53^{\prime \prime} \mathrm{N}$, long. $104^{\circ} 7^{\prime} 53^{\prime \prime} \mathrm{E}$ ). At the site, the average elevation is $2200 \mathrm{~m}$, the annual average temperature is $11.5^{\circ} \mathrm{C}$, and the annual temperature difference is small. In summer, the average temperature is $18^{\circ} \mathrm{C}$, and that in winter is $7^{\circ} \mathrm{C}$. The day-night temperature difference is large. Rainfall is abundant, with an average rainfall of $909 \mathrm{~mm}$. The illumination is sufficient, with an annual sunshine duration of $1812 \mathrm{~h}$. The frost-free period is $180 \mathrm{~d}$. The climate is a subtropical monsoon humid climate with warm temperatures in winter, cool temperatures in summer, and a short spring and autumn with a long winter and summer. The soil is yellow soil with medium fertility. The plants were subjected to routine water and fertilizer management.

Opening process and phenology of individual flowers. Four trees in the growth stage without diseases or insect pests were randomly selected from different slopes of the experimental forest, and 15 flowers were randomly marked. The opening process and the numbers of flowers in different periods were observed beginning at 8:30 in the morning. The morphology, petal changes, and anther color changes of the flowers were recorded.

To understand flowering dynamics, the flowering phenology of $C$. weiningensis was observed for 3 consecutive years from 2017 to 2019 , and the three phenophases (i.e., the early flowering stage, full flowering stage, and final flowering stage) were recorded. These phenophases were defined as follows: early flowering stage, $5 \%$ to $10 \%$ of the plants in bloom; full flowering stage, more than $30 \%$ of the plants in bloom; and final flowering stage, more than $95 \%$ of the plants withering (Zhu, 2021).

Floral organ morphology. Five C. weiningensis plants were selected, and 15 flowers were selected from each plant. The petal length and width, corolla diameter, filament length, style length, stamen length, stamen number, ovule number, sepal number, and petal number of each flower at the full flowering stage were measured with a Vernier caliper (a dissecting microscope was also necessary). Average values were obtained. In addition, the CV was calculated with Excel software.

Pollen activity. Mature buds were collected before blossoming and transported back to the laboratory. They were inserted into flower mud and cultured with nutrient solution. Appropriate amounts of pollen at the early flowering stage (not yet blooming; petals wrapping the stamens) and at the full flowering stage (petals completely opened, stamens and pistils separated) were collected and carefully spread on glass slides with a brush, and one to two drops of $0.5 \%$ TTC solution were applied evenly. Then, the pollen was covered with a glass slide and cultured at $25^{\circ} \mathrm{C}$ for 15 to $30 \mathrm{~min}$. The pollen was counted under a microscope (magnitude, $10 \times 10)$.

Pollen with strong vitality appeared red or pink, and pollen with weak or no vitality was colorless or retained the original yellow color.

For each slide, five visual fields containing no fewer than 50 scattered pollen grains were selected. The calculation formula was as follows: $\mathrm{R}=(\mathrm{M} 1 / \mathrm{M} 2) \times 100 \%$, where $\mathrm{R}$ is the pollen viability (\%), M1 is the number of red or pink pollen grains with strong vitality in the visual field, and M2 is the total number of pollen grains with strong vitality, weak vitality, or no vitality.

Pollen germination rate. The pollen germination rate of $C$. weiningensis was determined by germination on solid medium at the full flowering stage. The pollen germination rate is affected by the concentration of sucrose. Therefore, under constant agar and boric acid concentrations, three media with sucrose concentrations of 50, 100, and 150 $\mathrm{g} / \mathrm{L}$ were used. Two or three droplets of medium were applied on a flat glass slide with a glass rod. After cooling, the surface of the medium was flattened with a cover glass slide (the medium had an appropriate thickness and a round shape). Then, the pollen was carefully collected with a brush and evenly placed on the surface of the medium. The pollen was placed into a culture dish with filter paper, and two to three drops of water were applied. The pollen was cultured at $25^{\circ} \mathrm{C}$ for $4 \mathrm{~h}$. Pollen germination was observed under a microscope (DM2500; Leica, Wetzlar, Germany). Germination was scored according to the criterion that the length of the pollen tube exceeded the diameter of the pollen grain. The pollen germination rate was calculated as follows: $\mathrm{R}=(\mathrm{x} / \mathrm{y}) \times 100 \%$, where $\mathrm{R}$ is the pollen germination rate $(\%), \mathrm{x}$ is the number of germinated pollen grains in the field of vision, and $\mathrm{y}$ is the total number of pollen grains (germinated and ungerminated).

Stigma receptivity. Stigma receptivity was determined by the benzidine-hydrogen peroxide method (Dafni, 1992). Flowers that entered the full flowering stage at the same time were selected and labeled. Their stigma receptivity was measured every day until the stigma had no vitality. Every day, pistils were removed, their ovaries were extracted, and the section from the stigma to the base of the style was placed into the concave part of a glass slide. The stigma was soaked completely with benzidine-hydrogen peroxide reaction solution ( $1 \%$ benzidine:3\% hydrogen peroxide:water = $4: 11: 22$ ). If the reaction solution around the stigma was blue and was accompanied by a large number of bubbles, the stigma was considered receptive. According to the number of bubbles, the receptivity of the stigma was determined. The stigma was observed with a stereomicroscope, and photos were taken.

Pollen quantity and the $P / O$ ratio. Ten mature, intact stamens at the yellow bud stage were randomly collected. The anthers were carefully removed with tweezers and then put into a clean $1.5-\mathrm{mL}$ centrifuge tube. The anthers were dried in a $25^{\circ} \mathrm{C}$ oven until they burst and released pollen. To each centrifuge tube, $1 \mathrm{~mL}$ of $1 \%$ pectinase solution was added. The anther sample was fully oscillated for $24 \mathrm{~h}$ to release all the pollen from the anther. A suspension of pollen grains was prepared and evenly distributed in the solution. Immediately, $1 \mu \mathrm{L}$ of the solution was dropped onto a slide with a 5- $\mu \mathrm{L}$ pipette and then covered with cover glass. The pollen number was determined under a microscope (Leica DM2500). The average of three replicates was determined. The $\mathrm{P} / \mathrm{O}$ ratio of each flower was obtained by dividing the total pollen grain number by the number of ovules. The formula for calculating the pollen quantity in an anther was as follows: $M=(m \times 200) / 10$, where $M$ is the number of pollen grains per anther and $\mathrm{m}$ is the total number of pollen grains on the slide.

Floral organ morphology. Fully blooming flowers at the full flowering stage were selected. Anthers that naturally released pollen within $24 \mathrm{~h}$ were carefully removed with tweezers. The style was cut off near the middle with a single blade under a dissecting microscope, with half of the anther connected to the stigma and the other half connected to the base of the style. Then, the ovary was carefully opened to expose the ovule. A single ovule was extracted with a dissecting needle under a dissecting microscope. The anther, style, stigma, and ovule were separately placed into clean 2-mL centrifuge tubes filled with $2.5 \%$ glutaraldehyde (prepared with $0.1 \mathrm{~mol} / \mathrm{L}$ phosphate buffer) and then fixed in a refrigerator at $4{ }^{\circ} \mathrm{C}$ for $2 \mathrm{~h}$. Afterward, each specimen was washed three to four times with phosphate buffer $(0.1 \mathrm{~mol} / \mathrm{L})$, with each wash lasting $10 \mathrm{~min}$. Then, it was fixed in $1 \%$ osmium tetroxide (prepared with $0.1 \mathrm{~mol} / \mathrm{L}$ phosphate buffer) at $4{ }^{\circ} \mathrm{C}$ for $2 \mathrm{~h}$. Each specimen was again washed three to four times 
with phosphate buffer $(0.1 \mathrm{~mol} / \mathrm{L})$, with each wash lasting $10 \mathrm{~min}$, followed by gradient dehydration with $30 \%, 50 \%, 70 \%, 80 \%, 90 \%$, $95 \%$, and $100 \%$ ethanol. It was then treated with $3: 1,2: 2$, and $1: 3$ ethanol:tert-butyl alcohol mixtures for $10 \mathrm{~min}$, followed by treatment with $100 \%$ tert-butyl alcohol for $10 \mathrm{~min}$. The processed specimens underwent freezedrying treatment. Each treated sample was pasted onto the sample table with conductive tape and then plated with gold (MSP-mini; IXRF Systema, Inc., Japan) for $30 \mathrm{~s}$. Then, the sample was observed and photographed with a scanning electron microscope (TM4000 Plus; Hitachi, Tokyo, Japan) under the conditions of $10 \mathrm{kV}$, high vacuum, and BSE electron mode.

Pollen tube germination. Beginning at 7:00 AM every day, the temperature was recorded every $2 \mathrm{~h}$ for 3 consecutive days, and the best pollination time was calculated according to the observation data.

At the full flowering stage, the anther of a plant whose pollen had not yet been released was collected. The anther was taken to the laboratory and baked under a heat lamp to release pollen. The temperature was maintained at $25^{\circ} \mathrm{C}$. Among the plants used for pollen collection, those with large differences in petals and color were selected for pollination. To guarantee pollen free from pollution, materials with unopened petals, intact anthers, and normally growing styles were collected for artificial pollination. After pollination, the flowers were bagged with sulfuric acid paper, tied tightly with iron wire, and labeled. After $7 \mathrm{~d}$, the iron wire and sulfuric acid paper were removed. Three healthy tree individuals were selected to repeat the experiment.

The pistils were collected $12,24,48$, and $72 \mathrm{~h}$ after pollination, with nonpollinated pistils collected as a control. The materials were fixed in a centrifuge tube containing Carnot's fixative, transferred to $70 \%$ ethanol, and stored in a refrigerator at $4{ }^{\circ} \mathrm{C}$. The pistils were taken from the fixative solution, and the style was cut off at the base. The style was soaked in $50 \%$ and $30 \%$ ethanol, washed with distilled water three times ( 10 min per wash), and then softened with $8 \mathrm{~mol} / \mathrm{L} \mathrm{NaOH}$ for $2.5 \mathrm{~h}$. Aniline blue $(0.1 \%)$ staining was performed for $5 \mathrm{~h}$. The style was flattened gently, pressed onto a slide, and covered with another slide. Pollen germination on the stigma and the time required for the pollen tube to reach the base of the style were determined under a fluorescence microscope (Leica DM3000).

\section{Results}

\section{Flowering phenophases}

The flowering biology of $C$. weiningensis was observed and recorded for 3 consecutive years from 2017 to 2019 (Table 1). In the middle of February, $C$. weiningensis began to sprout shoots and develop new leaves. The leaves were alternate, and the twigs were glabrous. Flower buds were mainly located at the top and in the axils of current-year twigs. The growth and development of the flower buds of $C$. weiningensis require a long time.

Table 1. Flowering times of Camellia weiningensis.

\begin{tabular}{|c|c|c|c|c|c|c|c|}
\hline \multirow{2}{*}{$\begin{array}{l}\text { Observation } \\
\mathrm{yr}\end{array}$} & \multicolumn{2}{|c|}{ Early flowering stage } & \multicolumn{2}{|c|}{ Full flowering stage } & \multicolumn{2}{|c|}{ Late flowering state } & \multirow{2}{*}{$\begin{array}{c}\text { Whole flowering } \\
\text { stage }\end{array}$} \\
\hline & Start date & Days & State date & Days & State date & Days & \\
\hline 2017 & 7 Feb. 2017 & 17 & 24 Feb. 2017 & 20 & 16 Mar. 2017 & 16 & 53 \\
\hline 2018 & 11 Feb. 2018 & 12 & 27 Feb. 2018 & 18 & 17 Mar. 2018 & 19 & 49 \\
\hline 2019 & 14 Feb. 2019 & 15 & 1 Mar. 2019 & 14 & 15 Mar. 2019 & 24 & 53 \\
\hline
\end{tabular}

At the end of May, a small number of trees exhibited flower bud differentiation. In the middle of June, almost all of them entered the stage of flower bud differentiation. Afterward, the flower buds gradually extended horizontally and vertically, and the flower buds could be distinguished from the leaf buds with the naked eye. The development of flower buds ended just before flowering. Some flower buds entered the budding stage in the middle of January (Fig. 1A). On a single plant, flower buds of different sizes appeared, and two or three flowers were scattered on the plant. In early February, $C$. weiningensis entered the early flowering period (Fig. 1B). This stage lasted a long time (about half a month). Approximately $5 \%$ of the flowers on the whole plant were completely open at a given time, with a large number of buds. At this time, the diameter and length of the buds were larger than those of the buds in the budding period. The petals gradually loosened, preparing for the arrival of the full flowering period. With increasing temperatures, the full flowering period (Fig. 1C) occurred from the end of February to the beginning of March, when more than $95 \%$ of the flowers were in full bloom. This stage, which lasted $\approx 17 \mathrm{~d}$, was characterized by the largest flower diameters and the largest number of flowers and thus was when the largest number of insects were attracted. The longer the flowering period, the more insects were attracted, which, in turn, was more conducive to the spread of pollen. After the full flowering period, the late flowering period, which lasted $\approx 20 \mathrm{~d}$, took place (Fig. 1D). During this period, the flowers gradually withered. The entire flowering period of $C$. weiningensis lasted $\approx 52 \mathrm{~d}$. During the dynamic flowering period, flowering
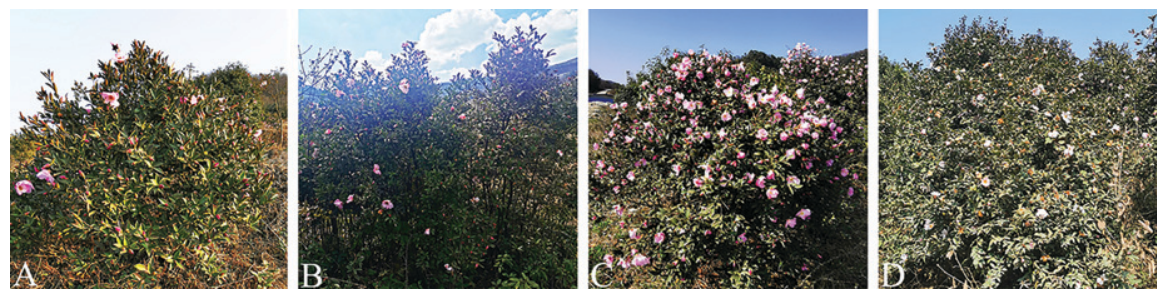

Fig. 1. Flowering phenology of Camellia weiningensis. (A) Budding stage. (B) Early flowering stage. (C) Full flowering stage. (D) Late flowering stage.

Table 2. Flowering processes of individual flowers of Camellia weiningensis.

\begin{tabular}{lccccc}
\hline Serial no. & Beak-breaking $(\mathrm{d})$ & Flowering $(\mathrm{d})$ & Blooming $(\mathrm{d})$ & Withering $(\mathrm{d})$ & Total $(\mathrm{d})$ \\
\hline 1 & 3 & 3 & 5 & 2 & 13 \\
2 & 2 & 3 & 4 & 3 & 12 \\
3 & 4 & 2 & 4 & 2 & 12 \\
4 & 2 & 4 & 3 & 4 & 13 \\
\hline
\end{tabular}

and flower fall occurred, along with the growth and development of young fruits. The flowering time was somewhat variable among plants and years, which may have been due to different climate conditions. In addition, the duration of flowering was also variable, but this variation was small.

Individual-flower opening process. The opening processes of individual flowers on different $C$. weiningensis plants were basically the same. According to the morphological characteristics of flowers, the flowering process of an individual flower can be divided into four stages (Table 2).

(1) Beak-breaking stage (Fig. 2A1 and B1). Flower buds expand, and the bracts gradually begin to loosen. The pink portion of the petals is exposed. The top of the flower appears bluish brown. The petals wrap the mens tightly. The anthers are light yellow, the stigma is light green, and three or two cracks converge. The flower buds continue to grow, and the petals are slowly loosened. his stage lasts 2 to $3 \mathrm{~d}$.

(2) Flowering stage (Fig. 2A2, A3, B2, and $\mathrm{B} 3$ ). The duration of this stage is $\approx 3$ to $4 \mathrm{~d}$. The petals continue to extend horizontally and longitudinally. They are slightly open (but not fully open), and the color remains pink. Compared with the petals at the beak-breaking stage, those at this stage exhibit an increased diameter. The stamens and pistils gradually separate, the style color remains unchanged, the stigma turns up slightly, and the anthers are round, full, and yellow. At this stage, the anthers remain in a state in which pollen is not released. A light aroma is released during this opening stage.

(3) Blooming period (Fig. 2A4, A5, B4, B5). The perianth and petals are fully opened, 

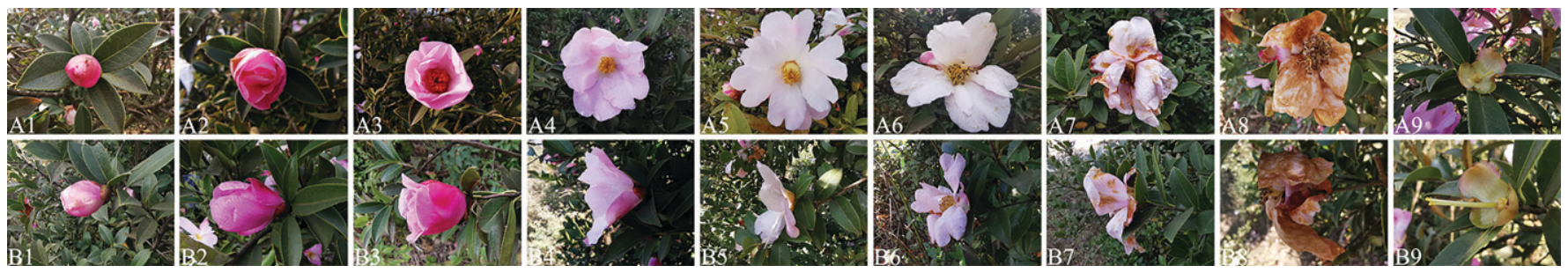

Fig. 2. Flowering process of individual flowers of Camellia weiningensis. (A1) Front view at the beak-breaking stage. (B1) Lateral view at the beak-breaking stage. (A2 and A3) Front view at the flowering stage. (B2 and B3) Lateral view at the flowering stage. (A4 and A5) Front view at the blooming stage. (B4 and B5) Lateral view at the blooming stage. (A6-A9) Front view at the withering stage. (B6-B9) Lateral view at the withering stage.

with the flower diameter reaching the maximum. The petals gradually turn from pink to light pink. The pistils and stamens are completely separated, and the stamens are radiated. The anthers appear bright yellow and gradually dehiscent to release pollen. The stigma continues to elongate and divide, the two- or three-lobed stigma continues to roll up, and the lobes separate from each other. Pollen is scattered on the stigma and begins to germinate. The style is light green, secreting mucus, and accepting pollen. The whole flower emits a strong fragrance and attracts insects. This stage lasts for 3 to $5 \mathrm{~d}$.

(4) Withering period (Fig. 2A6-A9 amd B6-B9). The withering period begins soon after flowering. The diameters of the flowers gradually decrease, and the petals turn from pink to brown. At the early stage of withering, the petals roll down and back, and the color of the petals does not change. The anthers contract and shrivel, and the color changes from bright yellow to dark yellow. The stamen and petal fall off at the same time. Although the pistil does not fall off, the stigma gradually shrinks, and its color gradually deepens from light green to dark brown.

The individual-flower opening time of $C$. weiningensis is 12 to $13 \mathrm{~d}$. During the full

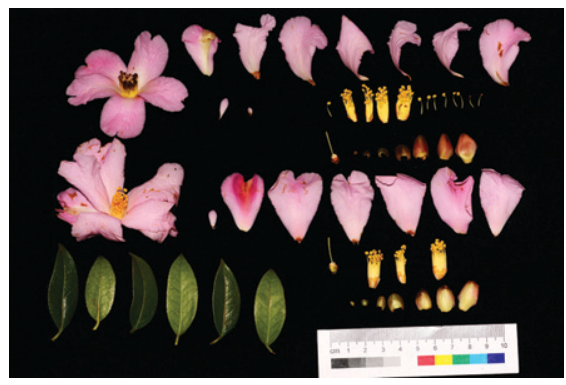

Fig. 3. Floral morphology of Camellia weiningensis. flowering period, the observation of 15 marked flowers showed that the perianth components of the flower gradually opened at $\approx 830 \mathrm{HR}$, and the petals opened at $1030 \mathrm{HR}$, with a small number of petals fully opened. From 1230 to $1630 \mathrm{HR}$, most flowers blossomed, and all flowers finished blooming at $1830 \mathrm{HR}$ in the afternoon.

Floral morphology. The morphological characteristics of the flowers of $C$. weiningensis were observed (Fig. 3). C. weiningensis produces complete flowers composed of a petiole, receptacle, calyx, corolla, stamen group, and pistil group. Usually, one to three flowers grow on the top of the branch or in the axils of the leaves. The flowers are bisexual, including both pistils and stamens. According to Table 2, the number of sepals was four to seven. They were $14.6 \mathrm{~mm}$ long, $12.2 \mathrm{~mm}$ wide, green or grayish purple, elliptic, smooth, and glabrous. There were six to eight petals, which were separated, different in size (46.8 $\mathrm{mm}$ in length and $34 \mathrm{~mm}$ in width) and bright in color. The petal base and stamen were connected, and the anther and filament formed the stamen. The stamens formed many whorls. Those in the inner whorl were $13.4 \mathrm{~mm}$ long, and those in the outer whorl were $24.6 \mathrm{~mm}$ long. Each flower had 106 stamens on average. The pistil was composed of the ovaries, style, and stigma. The style was two- to three-lobed, with an average length of $16.6 \mathrm{~mm}$, which was greater than the length of the inner-whorl stamens but shorter than that of the outer-whorl stamens. The stigma rolled back. The ovary, which is a superior ovary, contained three to five chambers and was densely lined with fine white hairs. In the chamber of the ovary, there were three to four inverted ovules, with an average of 10 ovules in each ovary. The flowers of $C$. weiningensis were large, with an average diameter of $83 \mathrm{~mm}$ in full bloom, and the corolla was cross-shaped.

The coefficients of variation of the floral organ morphological characteristics of $C$. weiningensis were $6.77 \%$ to $23.18 \%$ (Table 3 ), with that of the sepals being the largest and that of the stamens being the smallest (as well as the most stable). Except for that of sepals, the $\mathrm{CV}$ of the floral organ indexes were less than $20 \%$, and those of the number of petals and number of stamens were both less than $10 \%$. Therefore, the variation in the quantitative characteristics of the floral organs of $C$. weiningensis was low.

The upper part of the style connects to the stigma, and the lower part connects to the ovary. The pollen tube enters the ovary through the style. According to SEM, the plant surface was glabrous but contained holes arranged in a honeycomb-like pattern. The edge gap is arranged loosely, and the center gap is arranged tightly. There is an obvious difference between the outer epidermis and the inner epidermis. The stigma grows gradually and expands and branches, and the tip curls outward from the center, showing a "Y" shape. The pollen surface is two- to three-lobed, which increases the stigma contact area. This maximizes the acceptance of pollen released during anther dehiscence and improves the self-pollination ability and reproduction of the plant. The contact surface is covered with papillary cells, which have the same morphological characteristics. The cells are strip-shaped and densely arranged. The stigma of $C$. weiningensis is wet, with abundant secretory fluid on the surface and between papillary cells. The surface is plump, which facilitates pollen adhesion, thereby providing a suitable germination environment for pollen (Fig. 4A and B). The differentiation between the style and the stigma is noticeable. The style is slender, and the stigma differentiates markedly at the upper

Table 3. Floral organs of Camellia weiningensis.

\begin{tabular}{|c|c|c|c|c|c|c|c|c|}
\hline Serial no. & Outer stamen length & Inner stamen length & Style length & Corolla diam & Sepal length & Sepal width & Petal length & Petal width \\
\hline 1 & 28 & 17 & 20 & 103 & 16 & 14 & 52 & 41 \\
\hline 2 & 26 & 11 & 17 & 92 & 14 & 13 & 41 & 30 \\
\hline 3 & 22 & 14 & 16 & 72 & 13 & 11 & 41 & 32 \\
\hline 4 & 19 & 11 & 12 & 73 & 13 & 10 & 39 & 29 \\
\hline 5 & 28 & 14 & 18 & 75 & 17 & 13 & 61 & 38 \\
\hline Total value & 123 & 67 & 83 & 415 & 73 & 61 & 234 & 170 \\
\hline Average & 24.6 & 13.4 & 16.6 & 83 & 14.6 & 12.2 & 46.8 & 34 \\
\hline Standard error & 3.56 & 2.24 & 2.65 & 12.38 & 1.62 & 1.47 & 8.44 & 4.69 \\
\hline CV $(\%)$ & 14.47 & 16.72 & 15.96 & 14.92 & 11.10 & 12.05 & 18.03 & 13.79 \\
\hline
\end{tabular}



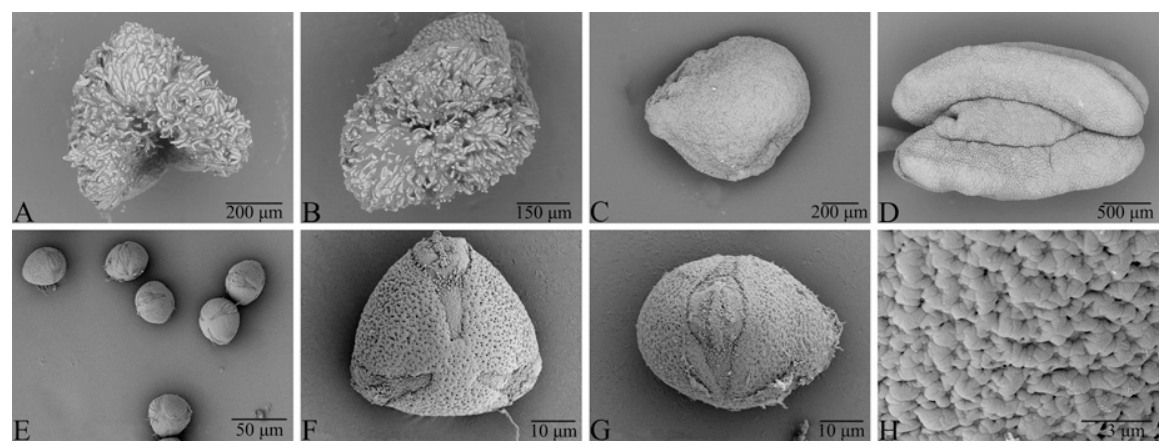

Fig. 4. The stigma, style, and ovule of Camellia weiningensis under a scanning electron microscope. (A and B) Stigma. (C) Ovule. (D) Anther. (E) Clustered pollen. (F) Polar view of the pollen. (G) Pollen germinating groove. (H) Pollen surface ornamentation. end of the style. Ovules are embedded in the ovary and are attached to the placenta. Ovules give rise to seeds. The ovule is spherical with a concave side. Its surface appears regularly wavy, with closely arranged cells (Fig. 4C). The ovule is inverted, and the micropyle parallels the funicle.

The morphologies of the anther and pollen were observed. The anther sacs are symmetrical on the left and right sides of the anther connections. The whole anther is butterfly-shaped, with numerous convex rhombi on the surface (Fig. 4D). The pollen of $C$. weiningensis is medium-sized. It is radially or hierarchically symmetrical and has a rhomboid cone shape. Under normal conditions, there are three germination pores (tremoids). The groove of the germination pore is slender and extends to the two poles, nearly reaching them. The pollen is spherical in equatorial view and trilobate in polar view. When the pollen grain begins to germinate, the three grooves protrude from the middle to the top and widen gradually (Fig. 4E and F). The pollen grain develops gradually from a rhombohedral cone shape to a spherical shape (Fig. $4 \mathrm{G})$. The outer surface of the pollen grain is uneven and smooth, and the ornamentation consists of net ridges and shallow grooves or holes. Irregular wavy ridges form a wrinkled line pattern, and the network of holes and reticular ridges in regular arrangement form a reticular line pattern. There are also striations similar to those in the brain (Fig. $4 \mathrm{H}$ ).

\section{Pollen vitality}

The TTC method was used to determine the pollen vitality of $C$. weiningensis at the beak-breaking stage and the blooming stage. There were significant differences in pollen vitality between these stages of flower development. The pollen vitality of $C$. weiningensis in the beak-breaking stage (Fig. 5A1) was $38 \%$, and that in the full flowering stage (Fig. 5A2) was $62 \%(P<0.05$; Table 4$)$. Because the vitality of pollen is higher in the full flowering stage, pollen produced at this stage has a better chance of successful pollination.

Sucrose is an indispensable nutrient for pollen culture in vitro. It not only plays a particularly important role in the processes of pollen germination and pollen tube wall synthesis but also provides nutrients for pollen germination and regulates external osmotic pressure. Different sucrose concentrations have different effects on the pollen germination rate of C. weiningensis. With an increase in the sucrose concentration, the pollen germination rate of $C$. weiningensis showed an upward trend (Fig. 5B1-B3, Table 5). When the sucrose concentration was $100 \mathrm{~g} / \mathrm{L}$, the germination rate reached a maximum of $54 \%$. When the sucrose concentration was $150 \mathrm{~g} / \mathrm{L}$, the pollen germination rate was $47 \%$. Therefore, the optimal sucrose concentration for $C$. weiningensis pollen germination was $100 \mathrm{~g} / \mathrm{L}$, at which the pollen germination rate was the highest, although no significant differences were observed among the three groups.

\section{Stigma receptivity}

The stigma receptivity of $C$. weiningensis at different flowering times (1-8 d) was detected with a benzidine-hydrogen peroxide reaction solution. According to the color change of the reaction solution and the number of bubbles around the stigma (Fig. 6, Table 6), the stigma of $C$. weiningensis exhibited receptivity from the first day to the fifth day of flowering. The activity of peroxidase varied among time periods. On the first day of flowering, the stigma had not yet opened (Fig. 6A1). The reaction solution around the stigma turned blue, with a small number of bubbles. On the second and third days of flowering, receptivity was the highest, and peroxidase activity reached a maximum (Fig. 6A2 and A3, respectively). The reaction solution around the stigma appeared blue, with a larger number of bubbles than that observed at the other flowering times. On the fourth day, the receptivity began to decline, and peroxidase activity weakened (Fig. 6A4 and A5). The reaction solution around the stigma remained blue, but

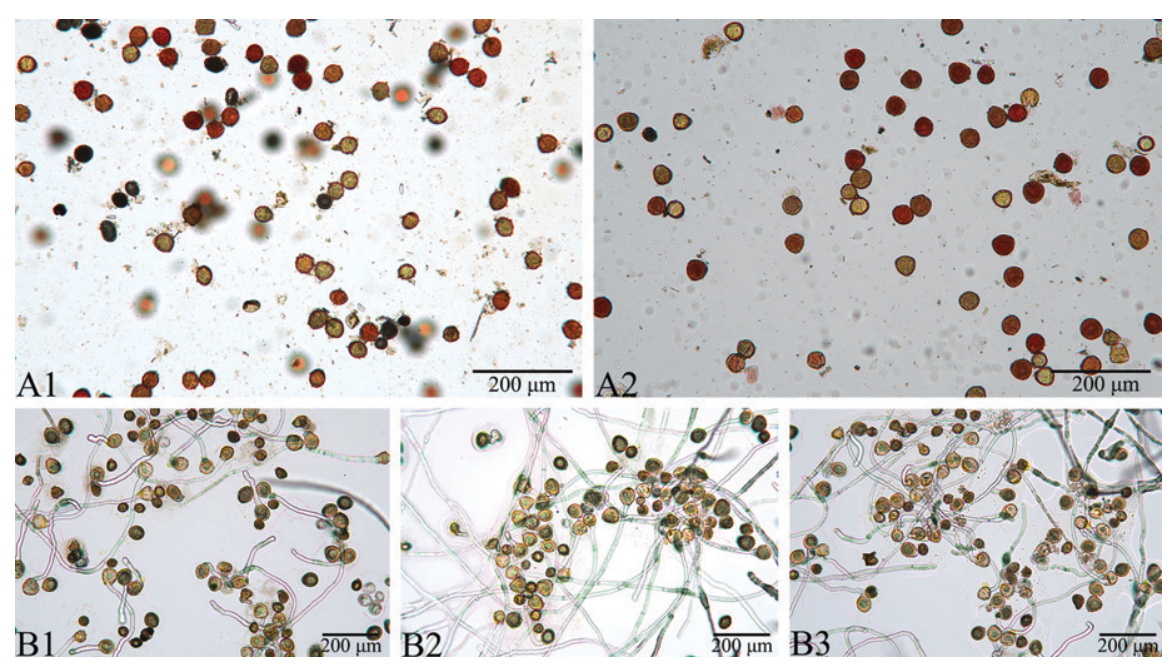

Fig. 5. Pollen vitality of Camellia weiningensis. (A1) Pollen at the beak-breaking stage. (A2) Pollen at the blooming stage. (B1) Pollen cultured with $50 \mathrm{~g} / \mathrm{L}$ sucrose. (B2) Pollen cultured with $100 \mathrm{~g} / \mathrm{L}$ sucrose. (B3) Pollen cultured with $150 \mathrm{~g} / \mathrm{L}$ sucrose.

Table 4. Pollen vitality in Camellia weiningensis at different flowering stages.

\begin{tabular}{lccc} 
Stage & Number of stained pollen grains & Total number of pollen grains & Pollen vitality (\%) \\
\hline Beak-breaking & 170 & 447 & $38 \mathrm{a}$ \\
Blooming & 189 & 305 & $62 \mathrm{~b}$
\end{tabular}

Different letters indicate significant differences according to the paired $t$ test.

Table 5. Pollen germination rates in Camellia weiningensis.

\begin{tabular}{lcccc}
\hline Serial no. & Agar $(\mathrm{g} / \mathrm{L})$ & Boric acid $(\mathrm{g} / \mathrm{L})$ & Sucrose $(\mathrm{g} / \mathrm{L})$ & Germination rate $(\%)$ \\
\hline 1 & 10 & 0.1 & 50 & 39 a \\
2 & 10 & 0.1 & 100 & $54 \mathrm{a}$ \\
3 & 10 & 0.1 & 150 & $47 \mathrm{a}$ \\
\hline
\end{tabular}

The same letter indicates no significant differences among the groups according to analysis of variance. 

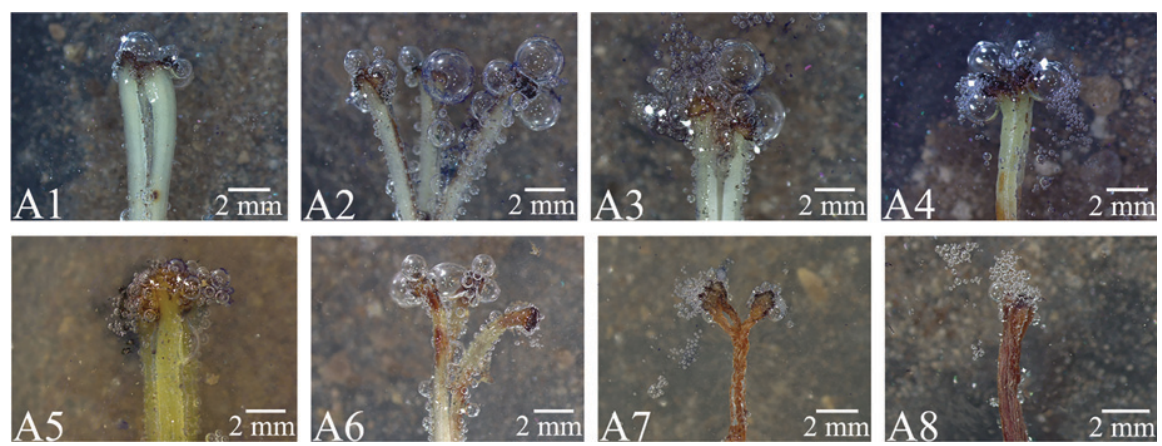

Fig. 6. Stigma receptivity of Camellia weiningensis according to benzidine-hydrogen peroxide. (A1) On the first day. (A2) On the second day. (A3) On the third day. (A4) On the fourth day. (A5) On the fifth day. (A6) On the sixth day. (A7) On the seventh day. (A8) On the eighth day.

Table 6. Stigma receptivity in Camellia weiningensis at different flowering times.

\begin{tabular}{lccc}
\hline Florescence & Depth of coloration (blue) & Bubble number & Receptivity \\
\hline Day 1 & +++ & +++ & +++ \\
Day 2 & ++++ & ++++ & ++++ \\
Day 3 & ++++ & ++++ & ++++ \\
Day 4 & +++ & +++ & +++ \\
Day 5 & ++ & ++ & ++ \\
Day 6 & - & + & + \\
Day 7 & - & - & - \\
Day 8 & - & - & - \\
\hline$++++=$ very strong receptivity; & $+++=$ strong receptivity; ++ & + \\
ity; $-=$ & &
\end{tabular}

the bubbles became smaller and fewer. On the sixth day, the stigma withered, and the top of the stigma turned black (Fig. 6A6). There was no blue reaction solution around the stigma, and the bubbles decreased. Thus, stigma receptivity was low. On the seventh and eighth days (Fig. 6A7 and A8, respectively), the stigma became nonreceptive. It withered and shrank. Color changes were not observed in the reaction solution, and no bubbles were produced.

\section{P/O ratio}

The test results of the $\mathrm{P} / \mathrm{O}$ ratio are summarized in Table 7 . The average number of pollen grains per anther was 2173 . The number of anthers per flower was $\approx 230,338$, and the average number of ovules per flower was 10. According to Cruden (1977), when the $\mathrm{P} / \mathrm{O}$ ratio is 18.1 to 39.0 , the breeding system is obligate autogamy; when the $\mathrm{P} / \mathrm{O}$ ratio is 31.9 to 396.0 , the breeding system is facultative autogamy; when the $\mathrm{P} / \mathrm{O}$ ratio is 244.7 to 2588.0, the breeding system is facultative xenogamy; and when the $\mathrm{P} / \mathrm{O}$ ratio is 2108.0 to $195,525.0$, the breeding system is obligate xenogamy. The $\mathrm{P} / \mathrm{O}$ ratio of $C$. weiningensis was 23,034 , which indicates that it is an obligate outcrosser.

\section{Fluorescence observation of pollen tube germination}

According to the temperature data collected from the test site for 3 consecutive d, $1500 \mathrm{HR}$, and the temperature difference between day and night was large (Fig. 7). The optimum pollination time was between 1300 and $1500 \mathrm{HR}$. the temperature peaked between 1300 and
The processes of pollen germination and pollen tube growth in nonpollinated $C$. weiningensis and outcrossing $C$. weiningensis were observed by fluorescence microscopy after 12 , 24,48 , and $72 \mathrm{~h}$, and the results are shown in Fig. 8. Compared with the control pollen, the outcrossed pollen could germinate and grow on the stigma and then enter the style smoothly. At $12 \mathrm{~h}$ before pollination, the pollen had germinated. At $12 \mathrm{~h}$ after pollination, the pollen tubes on both sides of the style had germinated together due to a hollow pollen tube. The tubes grew vertically downward. A single pollen tube on one side of the style emerged first and grew one-third of the way down the style. At $24 \mathrm{~h}$ after pollination, the pollen tube rapidly grew in a longitudinal direction. The number of germinated pollen grains and the length of the pollen tube increased significantly. A large number of pollen tubes grew halfway down the style, and the pollen tube on each side of the style grew slightly forward. At $48 \mathrm{~h}$ after pollination, the pollen tubes continued to grow downward and reached two-thirds of the way down the style. At $72 \mathrm{~h}$ after pollination, the pollen tubes gathered to one side of the style and reached the base of the style, with some passing through the base of the style.

\section{Discussion}

A comprehensive and systematic understanding of plant flowering biological characteristics is not only the premise of studying plant life history but also the basis for the study of the conservation, utilization, and breeding of germplasms (Shao et al., 2013). Research on flowering phenology in China started late and mainly focused on the relationships between flowering phenology, pollination mode, mating system, and environmental factors. The observational results of this study showed that the early flowering period of $C$. weiningensis is in mid-September, the initial flowering period is at the beginning of January, and the full flowering period is concentrated from February to March. At the full flowering stage, the number of flowers reaches the maximum, which prevents the influence of cold weather on floral organs. With an increase in temperature, insects become gradually active, which is beneficial to the spread of pollen. The opening process of flowers lasts 12 to $13 \mathrm{~d}$, and the flowering time is from 1030 to 1630 HR every day. The flowers open in the morning, bloom at noon, and slowly close at night. Wang et al. (2013) reported that the opening process of flowers of $C$. oleifera lasted 3 to $10 \mathrm{~d}$. Deng et al. (2009) reported that individual flowers lasted for 4 to $5 \mathrm{~d}$. Prolongation of the lifetime of a flower increases its probability of being pollinated, thereby guaranteeing reproduction and fruit setting. Therefore, the best pollination time of $C$. weiningensis is from the end of February to the beginning of March. According to a previous forestry study, flowering phenology is closely related to the fruit setting rate, fruit yield, and
Table 7. Pollen grain numbers in Camellia weiningensis.

\begin{tabular}{lcccccc}
\hline Species name & Replicate 1 & Replicate 2 & Replicate 3 & Avg & $\begin{array}{c}\text { Pollen grains } \\
\text { per flower }\end{array}$ & $\begin{array}{c}\text { Pollen/ovule } \\
\text { ratio }\end{array}$ \\
\hline C. weiningensis & 2,080 & 2,560 & 1,880 & 2,173 & 230,338 & 23,034 \\
\hline
\end{tabular}

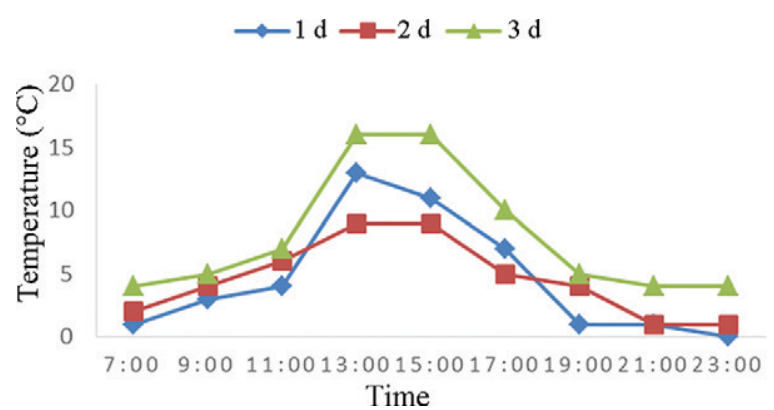

Fig. 7. Temperature at the experimental site at different times. 


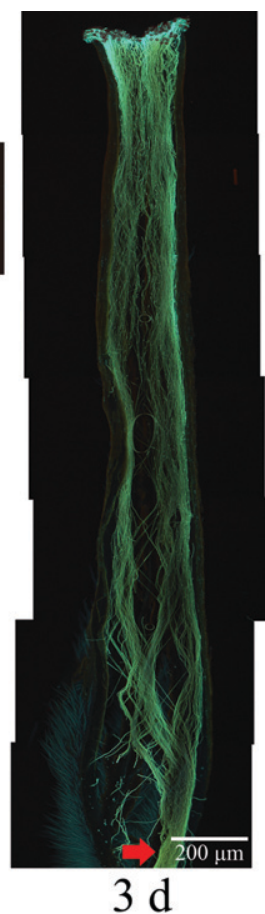

$2 \mathrm{~d}$

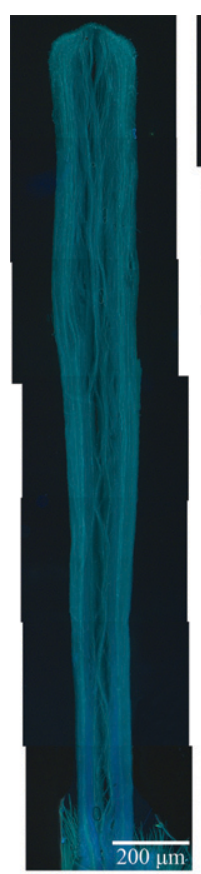

CK

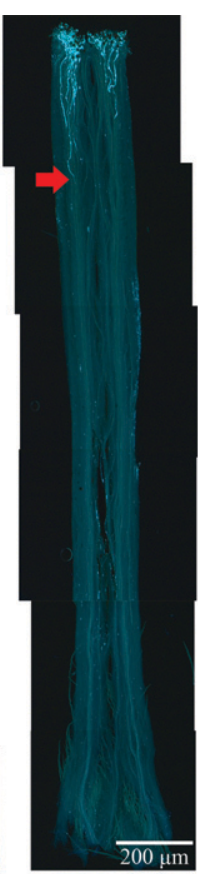

$12 \mathrm{~h}$

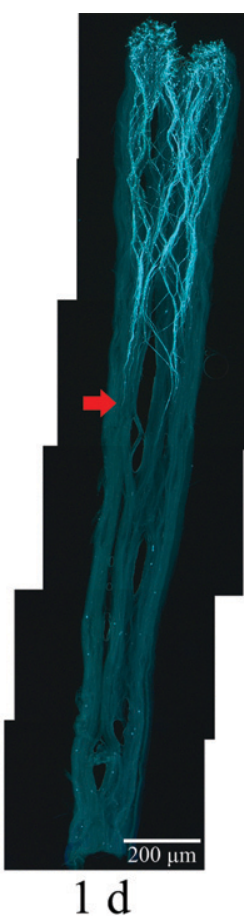

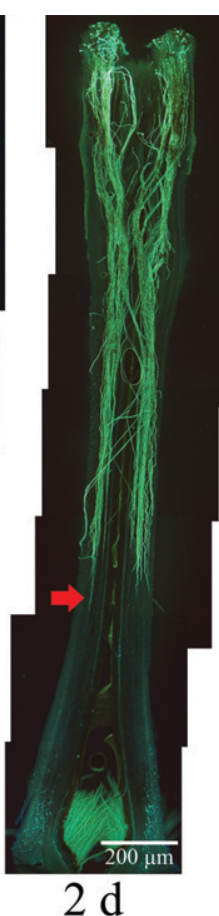

Fig. 8. Fluorescence observation of pollen tube germination in Camellia weiningensis.

fruit quality of $C$. oleifera. During the flowering period, low temperatures or high temperatures will affect the flowering, pollination, and fruit setting of the plant. According to research on Zanthoxylum bungeanum 'Lingshan', the flowering phenology affects the economic benefits of this plant. According to Chen et al. (2015), low temperatures in early spring delay the withering of rape flowers (Brassica campestris), and higher temperatures attract more insects to visit the flowers. Cold, frost, or frequent rainy days during the pollination period in early spring are very likely to cause pollination failure in $C$. weiningensis, which in turn results in a low seed-setting rate or even no fruit, even though the number of flowers is large. Our study revealed that the flowering pattern of $C$. weiningensis showed a normal distribution with a high peak, which is consistent with a "centralized flowering mode." This flowering pattern is the same as that of most plants ( $\mathrm{Li}$ et al., 2013; Schlindwein et al., 2014). There are two flowering patterns among individual plants: a large number of new flowers are produced every day or within a week (i.e., the centralized flowering mode), and a small number of new flowers are produced every day or several weeks (i.e., the "continuous flowering mode") (Javier, 1986; Okullo et al., 2004). After 3 consecutive years of observation (from 2017 to 2019), we found that the flowering phenology of $C$. weiningensis exhibited a normal distribution with a single peak. At the end of February and in early March, the centralized flowering mode, which is also found in many other plants (Javier, 1986), was observed. The concentrated flowering mode of Sapindus mukorossi allows it to avoid the effect of rainy weather from May to June on the frequency and efficiency of insect- aided pollination. To cope with extremely severe weather and to ensure successful pollination, Hypecoum erectum exhibits a concentrated flowering mode. The flowering mode of $C$. weiningensis involves the production of a large number of flowers within a short period. Such a flowering mode may allow a plant to avoid the influence of extreme cold weather in winter on flower organs and to attract more pollinators. A long flower life may serve as a strategy for adapting to a small number of pollinators.

The occurrence and arrangement of floral organs are of great significance in plant classification. They are closely related to fruit setting and fruit quality and can provide relevant information to reveal the systematic relationships of species groups (Tucker, 1996). Studies on the development of floral organs help clarify plant reproductive processes, which may lay a foundation for research on flowering management and cross-breeding. Comparisons of the flowering periods of the Cenruan series, the Xianglin series, the Gan series, and Guizhou Jiangdong 9 of C. oleifera show that although their early flowering stages are the same in terms of phenology, their remaining flowering stages differ because of differences in latitude, showing a normal distribution; thus, the flowering phenophase should be considered when selecting excellent Camellia reticulata plants (Yang et al., 2020). The flowers of $C$. weiningensis have six to eight petals, and the length and width of the petals are $4.68 \mathrm{~cm}$ and $3.4 \mathrm{~cm}$, respectively. The corolla diameter of the flowers is 7.2 to $10.3 \mathrm{~cm}$. According to Abe (2006), a flower with a diameter greater than $27.86 \mathrm{~mm}$ is considered large and can attract flower-visiting insects and increase pollination opportunities (Brunet and Sweet, 2006). Low temperatures affect not only the growth and development of plants but also the activity of pollinators, which then affects the reproduction of plants (Bingham and Orthner, 1998). In high-altitude areas, the heat affecting plants mainly depends on solar radiation (Rejšková et al., 2009). Because the corolla reflects solar radiation, the larger the corolla is, the more heat is concentrated in the reproductive organs of the flower. Therefore, the large corolla of $C$. weiningensis facilitates adaptation to low temperatures in mountainous areas. $C$. weiningensis releases a strong aroma during flowering. The floral fragrance of plants can attract pollinators to visit (Wright et al., 2002). The pollinators of C. weiningensis are mainly bees. The petals of $C$. weiningensis are pink and white, the stamens are yellow, the filaments are white, and the stigmas are yellow. Bees show affinity to white and yellow. Therefore, the flower color of $C$. weiningensis helps attract insects to stay on the stigma and stamens. The stamens of $C$. weiningensis are arranged in inner and outer rings. Generally, the stamens in the outer ring are $24.6 \mathrm{~mm}$ long, and those in the inner ring are $13.4 \mathrm{~mm}$ long. The number of stamens is $\approx 106$, with a small and stable $\mathrm{CV}$. These characteristics are representative of the primitive group of Camellia. Studies on the floral organs of different genera can provide evidence for reconstructing the evolutionary relationships between families and genera and assessing their evolutionary status.

Pollen is a reproductive organ with strong genetic conservatism that is not easily affected by the external environment. Its morphology and ornamentation are relatively stable and heritable. Pollen reflects evolutionary history to a large extent, and therefore, it is an important material for studying the origin, evolution, and genetic relationships of plants. Pollen can also be used to identify species and classify cultivars. The pollen grains of $C$. weiningensis are mainly rhombohedral in shape and medium-sized. The outer wall of the grain is mainly wrinkled and reticular, which are characteristics typical of Camellia pollen. Compared with its congener Camellia brevistyla, $C$. weiningensis exhibits a similar pollen morphology, but its pollen morphology is quite different from that of Camellia dedicata, Camellia albovillosa, and Sect. Theopsis. Pollen can be used as a morphological index for plant classification and cultivar identification and therefore is of great significance for studies of the relationships between genera and the genetic breeding of $C$. oleifera. The vitality and quantity of pollen grains are critical for plant reproductive abilities, directly affecting pollination, fertilization, and even the fruit setting rate of some plants. The vitality and quantity of pollen grains differ greatly among cultivars (Franchi et al., 2006; Sakata et al., 2000). In vitro culture of pollen is a common method for determining pollen vitality that is convenient and accurate. The composition of the medium used in this method affects the germination of pollen. The results of this study are consistent with the results 
obtained for Camellia chekiangoleosa (Ye et al., 2016) and Camellia chrysantha (Zhang et al., 2016) treated with different concentrations of sucrose. The germination rate of pollen increases with increasing sucrose concentrations; however, if the concentration is too high, the germination rate of pollen will be inhibited. Stigma receptivity is an important stage in the process of flower maturation and an important link in plant genetic breeding. It varies according to species and lasts from several hours to more than $10 \mathrm{~d}$ (Tao et al., 2010). In some plants, when the stigma is receptive, exudates and color changes at the top of the stigma and the opening of stigma lobes are observed (Galen and Plowright, 1987; Page et al., 2006). The stigma of $C$. weiningensis is wet, with secretions when receptive. The stigma is tow- to three-lobed, and the receptive period lasts 2 to $5 \mathrm{~d}$. The results of benzidine-hydrogen peroxide testing of the stigma in this study showed that this period facilitates later pollination; however, there were some human factors in this study that may affect interpretation. Whether the benzidine-hydrogen peroxide test result was consistent with the actual receptivity of the stigma remains to be verified by artificial pollination tests in different periods. According to the experimental results, $C$. weiningensis has a special heterozygous mating system, which is consistent with results reported in the literature (Gao and Yuan, 2017). According to Asghar et al. (2016), the growth and elongation of the pollen tube in the style channel can be observed more accurately and more clearly under a fluorescence microscope. According to the fluorescence microscopy observations in this study, the pollen tube of $C$. weiningensis has bright yellow fluorescence in the style channel. The more obvious the bright yellow fluorescence inside the wall during pollen germination is, the more accurate the estimate of pollen tube growth time will be. The influence of temperature on pollination experiments has been studied by forestry researchers in China. High temperatures promote the germination and growth of pollen tubes. The best pollination time of $C$. weiningensis is from 1300 to $1500 \mathrm{HR}$, which is the time of day with the highest temperature in the local area. Both internal and external factors affect pollen germination and pollen tube growth in $C$. weiningensis. The fluorescence observations in this study showed that the pollen tube reached the base of the style $72 \mathrm{~h}$ after pollination, which was different from the timing in $C$. oleifera (He, 2009; Liao et al., 2014). The reason for this phenomenon may be related to genes, nutrients, and environmental factors; however, the exact reason remains to be verified in the future.

\section{Literature Cited}

Abe, T. 2006. Threatened pollination systems in native flora of the Ogasawara (Bonin). Islands. Ann. Bot. 98:317-334, doi: 10.1093/aob/mcl117.

Ægisdóttir, H.H. and T.E. Thórhallsdóttir. 2006. Breeding system evolution in the Arctic: A comparative study of Campanula uniflora in Greenland and Iceland. Arct. Antarct. Alp. Res. 38: 305-312, doi: 10.1657/1523-0430(2006)38[305: BSEITA]2.0.CO;2.

Asghar, E., E. Ali, G. Akbar, and A. Roghaie. 2016. Comparison of different classical and molecular methods to identify superior self-compatible almond (Prunus dulcis Mill.) genotypes and evaluation of their traits. 91(1):36-42, doi: 10.1080/14620316.2015.1110990.

Barrett, S.C.H. 2003. Mating strategies in flowering plants: The outcrossing-selfing paradigm and beyond. Philos. Trans. R. Soc. Lond. B Biol. Sci. 358(1434):991-1004, doi: 10.1098/rstb.2003. 1301.

Bingham, R.A. and A.R. Orthner. 1998. Efficient pollination of al-pine plants. Nature 391:238 -239 , doi: $10.1038 / 34564$.

Brunet, J. and H.R. Sweet. 2006. Impact of insect pollinator group and floral display size on outcrossing rate. Evolution 60:234-246, doi: 10.1111/j.0014-3820.2006.tb01102.x.

Chen, F.J., W.N. Chen, and Y.H. Xie. 2015. Visiting behavior of Apis cerana on rape and characteristics of flowering and seed setting in early spring (in Chinese with an English abstract). Southwest China J. Agr. Sci. 28(02):498-502. <https://kns.cnki.net/kcms/ detail/detail.aspx?dbcode $=$ CJFD\&dbname $=$ CJF DLAST2015\&filename $=$ XNYX201502010\& $\mathrm{v}=\mathrm{e} 33$ Fosdi\%25mmd2FTGoC1LYxGpKDrLuqe 8ZjAgUZv03y65P1pVpc\%25mmd2BAcjEqKm1 GwsB $\% 25$ mmd2FIrs3b $>$.

Cruden, R.W. 1977. Pollen-ovule ratios: A conservative indicator of breeding systems in flowering plants. Evolution 31(1):32-46, doi: 10.1111/j.1558-5646.1977.tb00979.x.

Dafni, A. 1992. Pollination ecology: a practical approach. Oxford University Press, New York, doi: 10.1046/j.1420-9101.1993.6050776.x.

Deng, Y.Y., X.L. Yu, R.H. Rui, J. Huang, Y.F. $\mathrm{Xu}, \mathrm{W}$. Yang, and J.F. Xiang. 2009. Study on biological characteristics of Camellia Oleifera pollination (in Chinese). Non-wood Forest Res. 27(1):72-75. <https://www.docin.com/p-7717 35287.html $>$.

Duan, Y.W., Y.P. He, and J.Q. Liu. 2005. Reproductive ecology of the Qinghai-Tibet Plateau endemic Gentiana straminea (Gentianaceae), a hermaphrodite perennial characterized by herkogamy and dichogamy. Acta Oecol. 27:225232, doi: 10.1016/j.actao.2005.01.003.

Franchi, G.G., M. Nepi, M.L. Matthews, and E. Pacini. 2006. Anther opening, pollen biology and stigma receptivity in the long blooming species, Parietaria judaica L. (Urticaceae). Flora 202:118-127, doi: 10.1016/j.flora.2006.03.005.

Galen, C. and R.C. Plowright. 1987. Testing the accuracy of using peroxidase activity to indicate stigma receptivity. Can. J. Bot. 65(1):107-111, doi: 10.1139/b87-015.

Gao, C. and D.Y. Yuan. 2017. Observation system optimization and three-dimentional reconstruction of embryo sac in Camellia oleifera. J. Plant Physiol. J. 53(06):1104-1108. <https:// kns.cnki.net $/ \mathrm{kcms} /$ detail $/$ detail.aspx?dbcode $=\mathrm{CJF}$ D\&dbname $=$ CJFDLAST2017\&filename $=$ ZWSL $201706024 \& \mathrm{v}=\mathrm{cUfRLYFAyoV} \% 25 \mathrm{mmd} 2 \mathrm{Fwtk}$ 2KvPyOpUc2M2TLQIFQSwliWgYKQbK8\%25 mmd2BrASYKyLcX\%25mmd2B\%25mmd2BJo $5 \mathrm{~W} 2 \mathrm{VL}>$.

Harder, L.D. and S.C.H. Barrett. 1995. Mating cost of large floral displays in hermaphrodite plants. Nature 373(6514):512-515, doi: 10.1038/373 $512 \mathrm{a} 0$.

He, C.Y. 2009. The anatomical studies on the process of sexual reproduction of Camellia oleifera (in Chinese). Central South University of Forestry and Technology, Changsha, China,
Dissertation. $\quad<$ https://nxgp.cnki.net/kcms/ detail?v=3uoqIhG8C475KOm_zrgu4lQARvep2 SAkWGEmc0QetxDh64Dt3veMp-02F-tzNGH BIxiQDtQNqHPb4FgeKwY-oHy864hHUzpU\& uniplatform $=$ NZKPT $>$.

Javier, H. 1986. Flowering and fruiting phenology in the coastal shrublands of Doñana, south Spain. Plant Ecol. 68(2):91-98, doi: 10.1007/BF000 45059 .

Korner, C. 1999. Alpine plant life. Springer, Berlin. Kudo, G. and S. Suzuki. 2002. Relationships between flowering phenology and fruit-set of dwarf shrubs in alpine fellfields in northern Japan: A comparison with a subarctic heath-land in northern Sweden. Arct. Antarct. Alp. Res. 34:185-190, doi: $10.1080 / 15230430.2002 .1200$ 3483.

Li, Z.C., J. Li, H.Y. Lv, and M.H. Cao. 2013. Flowing phenology features of the rare desert plant Ammodendron argenteum[J]. Chinese J. Ecol. 32(11):2937-2943. <https://kns.cnki.net/ $\mathrm{kcms} /$ detail/detail. aspx ?dbcode $=$ CJFD\&dbname $=$ CJFD2013\&filename $=$ STXZ201311015\&v $=F$ W7Zs4nB8kSQmmIaF04pOGMhtBfRXb22a1U n4p131rCiq5U6aBEtOq91\%25mmd2Bhlk9rKW >.

Liao, T., D.Y. Yuan, C. Gao, Z. Feng, T. Jing, and X. Tan. 2014. Pollination, fertilization and early embryonic development of Camellia oleifera. Scientia Silvae Sinicae 50(02):50-55. <http://en. cnki.com.cn/Article_en/CJFDTOTAL-LYKE201 402008.htm>.

Mitchell, R.J., J.D. Karron, and K.G. Holmquist. 2004. The influence of Mimulus ringens floral display size on pollinator visitation patterns. Funct. Ecol. 18(1):116-121, doi: 10.1111/j. 1365-2435.2004.00812.x.

Molau, U. 1993. Relationships between flowering phenology and life history strategies in tundra plants. Arct. Alp. Res. 25:391-402, doi: $10.2307 / 1551922$.

Okullo, J.B.L., J.G. Hall, and J. Obua. 2004. Leafing, flowering and fruiting of Vitellaria paradoxa subsp. nilotica in savanna parklands in Uganda. Agrofor. Syst. 60:77-91, doi: 10.1023/B:AGFO. 0000009407.63892.99.

Page, T., G.M. Moore, J. Will, and G.M. Halloran. 2006. Onset and duration stigma receptivity in Kunzea pomifera (Myrtacese). Aust. J. Bot. 54:559-563, doi: 10.1071/BT05122.

Rejšková, A., J. Brom, J. Pokorný, and J. Korečko. 2009. Temperature distribution in light-coloured flowers and inflorescences of early spring temperate species measured by Infrared camera. Flora 205(4):282-289, doi: 10.1016/j. flora.2009.05.001.

Richards, A.J. 1986. Plant breeding systems[M]. Allen and Unwin Press, Winchester.

Sakata, T., H. Takahashi, I. Nishiyama, and A. Higashitani. 2000. Effects of high temperature on the development of pollen mother cells and microspores in barley Hordeum vulgare L. J. Plant Res. 113(4):395-402, doi: 10.1007/PL 00013947.

Shao, W.H., S.F. Diao, R.X. Dong, J.M. Jiang, and H.F. Yue. 2013. Study on geographic variation of morphology and economic character of fruit and seed of Sapindus mukorossi. For. Res. 26(05):/ 603-608. <http://en.cnki.com.cn/Article_enCJFD TOTAL-LYKX201305012.htm>.

Scheffknecht, S., S. Dullinger, G. Grabherr, and K. Hulber. 2007. Mating systems of snowbed plant species of the northeastern Calcareous Alps of Austria. Acta Oecol. 31(2):203-209, doi: 10.1016/j.actao.2006.09.001.

Schlindwein, C., C. Westerkamp, A.T. Carvalho, and P.M. Pinheiro. 2014. Visual signalling of nectar-offering flowers and specific morphological traits favour robust bee pollinators in the 
mass-flowering tree Handroanthus impetiginosus (Bignoniaceae). Bot. J. Linn. Soc. 176(3): 396-407, doi: 10.1111/boj.12212.

Tao, L., X.Y. He, S.B. Ni, L.L. Chen, and G.H. Sun. 2010. Flowering and pollinating characteristics of Macadamia. Chin. J. Trop. Crop 31(3):349-354. http://www.rdzwxb.com/EN/ Y2010/V31/I3/349.

Totland, Ø. 1997. Effects of flowering time and temperature on growth and reproduction in Leontodon autumnalis var. taraxaci, a late-flowering alpine plant. Arct. Alp. Res. 29:285-290, doi: $10.2307 / 1552142$.

Tsukaya, H., K. Fujikawa, and S.G. Wu. 2002. Thermal insulation and accumulation of heat in the downy inflorescences of Saussurea medusa (Asteraceae) at high elevation in Yun-nan, China. J. Plant Res. 115(4):263-268, doi: 10.1007/ s10265-002-0030-1.

Tucker, S.C. 1996. Diversity and evolutionary biology of tropical flowers. J. Evol. Biol. 9(1):120-124, doi: 10.1046/j.1420-9101.1996.9010120.x.

Wang, D.Z., W.X. Liang, and H.X. Huang. 1979. Camellia weiningensis $\mathrm{Y}$. K. Li-a newly discovered tea species in alpine mountainous area of Guizhou province. Guizhou For. Sci. Technol. (02):22-24. < https://kns.cnki.net/kcms/detail/ detail.aspx dbcode $=$ CJFD\&dbname $=$ CJFD7984 \&filename $=$ GZLY197902004\&v=fYVwv1iUo rATaFdaOxx2wMnPLpZ3MEgtvMHrTKF7f6 BEhEM2V8i9cmsXPMfZnYzY>.

Wang, X.N., Y.Z. Chen, R. Wang, S.F. Peng, L. Ma, L.S. Chen, W. Tang, and J. Luo. 2013.
Flowering and pollinating specifications of Camelia oleifera cultivars (in Chinese with an English abstract). J. Cent. South Univ. Forest. Technol. 33(12):1-61181. < https://kns.cnki. net $/ \mathrm{kcms} /$ detail/detail.aspx?dbcode $=\mathrm{CJFD} \&$ dbname $=$ CJFDHIS2 $\&$ filename $=$ ZNLB2 $01312001 \& \mathrm{v}=\mathrm{vwQtW}$ \% $\% 25 \mathrm{mmd} 2 \mathrm{BzDP} 4$ WrouCSOkCwkxzQrS2O8IcBiW7gATP04\% 25mmd2F6\%25mmd2B4V7f9UCAI2v WE7c1Gj>

Wright, G.A., B.D. Skinner, and B.H. Smith 2002. Ability of honeybee, Apis mellifera, to detect and discriminate odors of varieties of Canola (Brassica rapa and Brassica napus) and Snapdragon flowers (Antirrhinum majus). J. Chem. Ecol. 28(4):721-741, doi: 10.1023/A: 1015232608858 .

Wright, J.W. and T.R. Meagher. 2003. Pollination and seed predation drive flowering phenology in Silene latifolia (caryophyllaceae). Ecology 84(8):2062-2073, doi: 10.1890/02-0676.

Wyatt, R. 1982. Inflorescence architecture: How flower number, arrangement, and phenology affect pollination and fruit-set. Amer. J. Bot. 69(4):585-594, doi: 10.2307/2443068.

Yang, Z.P., Y. Xie, Y.Q. Cao, Z.Y. Xu, M.H. Cun, H.D. Ren, and E.X. Duan. 2020. Flowering phenophase of Camellia reticulata (in Chinese with an English abstract). Forest Inventory Plan. 45(05):19-25. <https://kns.cnki.net/KCMS/detail/ detail.aspx ?dbcode $=$ CJFQ \&dbname $=$ CJFDLAST 2020\&filename $=$ LDGH202005004\&v=MjI2NTE xVDNxVHJXTTFGckNVUjd1Z1lPZG9GaURn
VXJySktTbk1ackc0SE5ITXFvOUZZSVI4ZVg xTHV4WVM3RGg=>.

Ye, X., Y. Xie, and H.S. Ru. 2016. Pollen germination and storage of Camellia chekiangoleosa (in Chinese with an English abstract). J. Northwest Forest. Univ. 31(01):103-106 + 129 . $<$ https://kns.cnki.net/KCMS/detail/detail.aspx? dbcode $=$ CJFQ \&dbname $=$ CJFDLAST2016\& filename $=$ XBLX201601020\&v $=$ MjI1OTJUM 3FUcldNMUZyQ1VSN3VmWU9kb0ZpRGh VYnJBUFMvSGRyRzRIOWZNcm85SFpJU jhlWDFMdXhZUzdEaDE $=>$.

Zhang, P.X., B. Yu, J.F. Chen, C.Y. Zhao, C.P. Zou, and Y.B. Sun. 2016. Pollen germination and low temperature treatments of Camellia nitidissima (in Chinese with an English abstract). J. West China Forest. Sci. 45(02):94-97. $<$ https://kns.cnki.net/KCMS/detail/detail.aspx? dbcode $=$ CJFQ \& dbname $=$ CJFDLAST2016\& filename $=$ YNLK201602019\&v $=$ MzI0MzkxRn JDVVI3dWZZT2RvRmlEaFc3N05QQ1BIWm JHNEg5Zk1yWTIFYllSOGVYMUx1eFITN0R oMVQzcVRyV00 $=>$.

Zhu, X.J. 2021. The study flowering phenology of Armeniaca zhengheensis (in Chinese with an English abstract). J Fujian Forest. Sci. Technol. 48(01):50-53 + 111. <https://kns.cnki.net/KCMS/ detail/detail.aspx? dbcode $=$ CJFQ \&dbname $=$ CJFDL AST2021\&filename $=$ FJLK202101011\&v $=$ MTAz ODhITkRNcm85RVpZUjhlWDFMdXhZUzdEaD FUM3FUcldNMUZyQ1VSN3VmWU9kb0Z5bm tVcnJOSXImSFpiRzQ $=>$. 